\title{
Assessing the Relationship between Economic Factors and Adverse Events in an Active War Theater Using Fuzzy Inference System Approach
}

\author{
Erman Cakit, Member, IACSIT and Waldemar Karwowski
}

\begin{abstract}
The main purpose of this study was to investigate the relationship between adverse events and infrastructure development investments by estimating the number of adverse events in an active war theater based on the allocation of infrastructure development projects utilizing a fuzzy inference systems (FIS) approach. The considered model input variables included the total number of economic improvement projects and their associated budgets at different time periods in Afghanistan between 2002 and 2009. The output variables were the estimated numbers of people killed, wounded, and hijacked in different sectors of Afghanistan in 2010. Six different prediction models were developed and tested with an independent datasets. The prediction accuracy of each FIS model was evaluated and compared based on the mean absolute errors (MAE). It was concluded that the FIS is a useful modeling approach that can be applied under scenario-based conditions to support decision makers in analyzing historical economic data on how allocation of regional infrastructure development funds can best help reducing the onset of adverse events in an active war theater.
\end{abstract}

Index Terms-Adverse events, fuzzy inference system, human social cultural behavior (HSCB) modeling, infrastructure development.

\section{INTRODUCTION}

To protect civilians, today's military engages in irregular warfare where the civilian population is present [1]. The Department of Defense (DoD) describes irregular warfare as "a violent struggle among state and non-state actors for legitimacy and influence over the relevant population(s)" [2]. To address the challenges of irregular warfare, the Human Social Culture Behavior (HSCB) modeling program was initiated [3]. The main objective of HSCB program was to guide military in understanding different cultures while operating in overseas countries [4], and to improve organizing and controlling the human terrain during nonconventional warfare and other missions [5].

Nowadays, there is a need for HSCB models in military operations to understand the behavior and structure of organizational units at a macro level (economies, politics,

Manuscript received July 15, 2014; revised December 20, 2014. This study was supported in part by Grant No. [1052339], Complex Systems Engineering for Rapid Computational Socio-Cultural Network Analysis, from the Office of Naval Research. The authors acknowledge the helpful guidance of ONR program management, and the contributions of the technical team.

E. Cakit is with the Department of Industrial Engineering, Aksaray University, 68100, Aksaray, Turkey (e-mail: ermancakit@aksaray.edu.tr).

W. Karwowski is with the Department of Industrial Engineering and Management Systems, University of Central Florida, Florida, FL 32816-2450 USA (e-mail: wkar@ucf.edu). socio-cultural regions) and a micro level (social networks, tribes, military units) [6]. For instance, infrastructure development has been started during ongoing adverse events in Afghanistan [7]. Thus, HSCB models are attracting much attention with regard to current and future operational requirements.

Because an HSCB model can be defined as a complex system, computational modeling and simulation techniques are needed to deal with this complexity. While researchers in social science are contributing to HSCB modeling efforts by considering observations of human social behavior and communication [8], the program also needs the capability to represent variety of complex, ill-defined, and imprecise socio-economic-cultural concepts. Such a challenge can be met by application of fuzzy inference systems (FIS) modeling approach. To our best knowledge, there is currently no study that applies any soft-computing technique to investigate the relationship between adverse events and economic development projects in an active war theater.

This study investigates the application of Takagi-Sugeno-Kang (TSK) and the Mamdani fuzzy inference system (FIS) models to examine the relationship between adverse events and infrastructure development projects in Afghanistan, where the accuracy of the predictions is directly beneficial from an economic and humanistic point of view.

\section{BACKGROUND}

There are various challenges associated with problems related to representing social science data. Researchers have emphasized some of these difficulties. Numrich and Tolk [9] summarized these difficulties as lack of common vocabulary, variations in modeling approaches, and data acquisition. Similarly, Schmorrow et al. [10] highlighted the challenge of leveraging Modeling and Simulation (M\&S) for HSCB. The difficulties were summarized as the usage of M\&S tools within different complexity levels. To apply various methodologies in the domain of HSCB, such difficulties must be understood and specific modeling requirements must be met.

Studies in this area emerge from data-driven, statistical approaches where the modeler empirically derives the HSCB model from patterns identified in the data [11]. For example, terrorist attacks are not random in space and time, and as a result there are patterns that exist. It is possible to discover representative patterns in adverse activity or behavior over time and space by analyzing geospatial intelligence on various reported incidents, as stated by the Director of 
National Intelligence, Open Source Center (OCS) [12]. The OSC study of terrorism in Afghanistan highlighted the following types of analysis: mapping incident density, identifying the dominant ethnic group where incidents occurred, mapping incidents by district, mapping incidents by province, identifying the mean center of incidents over time, calculating the standard deviation (spatial pattern/trend) of overall incidents, mapping total incidents by month, and computing the mean center of incidents by month [13].

Similarly, there are a lot of challenges with HSCB modeling, particularly with regard to handling complexity, uncertainty, etc. Prediction is a particularly difficult task. The use of soft computing techniques with HSCB can mitigate these challenges. Fuzzy inference systems can be used for solving complex, ill-defined, and nonlinear problems including security and safety issues [14]-[16]. To demonstrate this, we're going to use FIS to analyze a certain characteristic we know aligns with stability and lack of violence (infrastructure \& economic spending) and see if we can use it to do a really difficult but useful modeling task: predict adverse events.

\section{Methodology}

\section{A. Dataset}

Two different kinds of Afghanistan datasets provided by the HSCB program management were utilized in this research, including adverse events and fourteen infrastructure development projects which took place between 2002 and 2010 (see Fig. 1). The adverse event dataset, which includes information regarding the date of event, incident type, number of people killed, wounded, and hijacked, province, city, district, description of the event, and simple event summary. The infrastructure aid dataset, which includes information regarding the province, city, district, project types, and allocated budget information for different sectors [17].

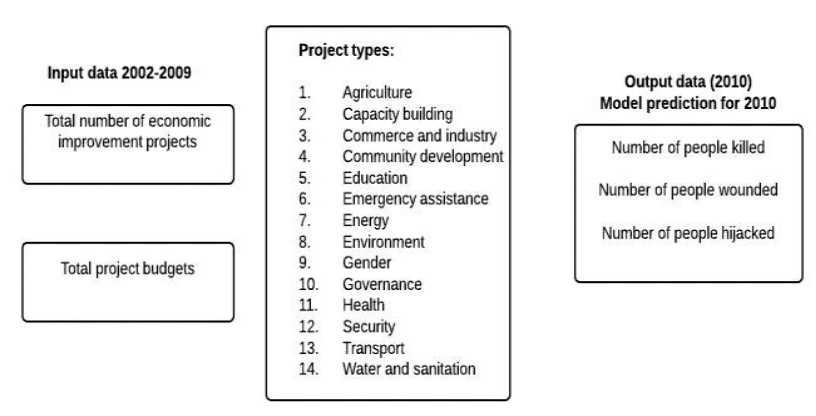

Fig. 1. Input and output data.

The total number of economic improvement projects and the total budget of those projects have been used to estimate the occurrence of adverse events. We considered a total of 3 outputs reflecting the adverse events in terms of the number of people killed, wounded, and hijacked. The data was grouped for generating rules and validation as follows: years between 2002 and 2009 (totally 38400 records for rule generation purpose), and year 2010 (totally 4800 records for validation).

\section{B. Fuzzy Inference System}

A fuzzy system consists of five functional blocks including fuzzification, database, rulebase, inference operations, and defuzzification (see Fig. 2). Moreover, input and output variables can be included.

A fuzzification interface transforms real numbers of input into fuzzy sets with linguistic values such as low, medium, and high. A database which determines the membership functions is applied in the fuzzy rules. A rule base has a number of fuzzy if-then rules that include all possible fuzzy relationships between inputs and outputs.

The inference engine helps to achieve inference operations on the rules. A defuzzification unit is used for converting fuzzy results into crisp values. There are two popular types of fuzzy inference systems including Mamdani and Takagi-Sugeno-Kang (TSK) models that have been widely used for solving problems in several applications such as decision analysis, expert systems, prediction, data classification, image processing, optimization, control and system identification [19], [20].

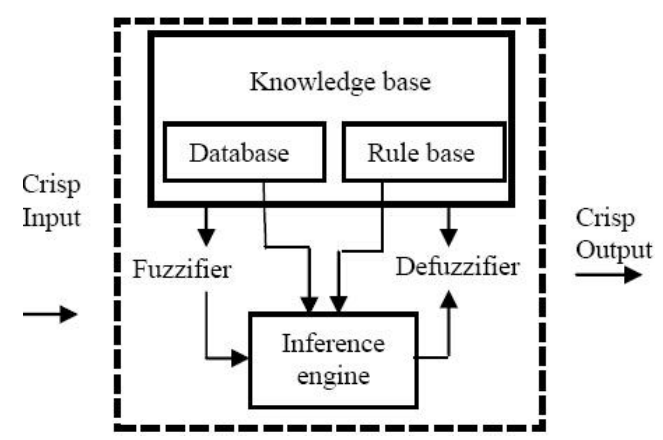

Fig. 2. Fuzzy inference system (Adapted from [18]).

Mamdani's FIS was proposed by Mamdani and Assilian (1975) and the TSK models were introduced by Takagi and Sugeno (1985). Since output values have to be fuzzy in Mamdani type, and crisp in TSK type; the main difference between these two types of models is in generating the crisp value. Mamdani type FIS applies defuzzification techniques, however the TSK type FIS applies a weighted average and weighted sum methods while calculating the crisp output

\section{Mamdani Fuzzy Model}

The Mamdani fuzzy model proposed by Mamdani and Assilian [21] was one of the first developed fuzzy set theory based control systems. The output membership functions of Mamdani model are fuzzy sets. After the aggregation process, defuzzification is necessary for each output variable to convert a fuzzy set to a crisp value.

A defuzzifier transforms the fuzzy results obtained from inference operations into a crisp output. There are several defuzzification methods such as centroid of area (COA), center of gravity, mean of the maximums, and smallest of the maximums. The most common defuzzifization method is the COA (Equation (1)).

$$
y_{C O A}=\frac{\int_{Y} \mu_{A}(y) y d y}{\int_{Y} \mu_{A}(y) d y}
$$

where $y_{C O A}$ is the crisp value for the z output and $\mu_{A}(y)$ is the summation of output membership function [22].

The Mamdani fuzzy model is the most popular fuzzy 
methodology which has been applied widely for several problems. Some of the advantages of Mamdani model are its suitability and intuitive for expert opinion, and that it is widely accepted.

\section{Takagi-Sugeno-Kang (TSK) Fuzzy Model}

The Takagi-Sugeno-Kang (TSK) fuzzy model was introduced by Takagi and Sugeno [23] in order to establish a systematic approach to construct fuzzy rules from a given input-output dataset [22]. There are similarities to the Mamdani method in several ways. For instance, the first two sections of the fuzzy inference systems (input fuzzification and applying the fuzzy operator) are completely same. The main difference is in the last part. The output membership functions can be a linear or constant for TSK model. A common rule in a Sugeno fuzzy model can be defined as following:

If $x$ is $\mathrm{A}$ and $y$ is $\mathrm{B}$ then $z=p x+q y+r$.

where $A$ and $B$ are fuzzy sets and $z$ is a non-fuzzy function. For a zero-order Sugeno model, the output level $z$ is a constant $(p=q=0)$. Many different resources are available in the literature for further explanations of FIS [15], [18], [19].

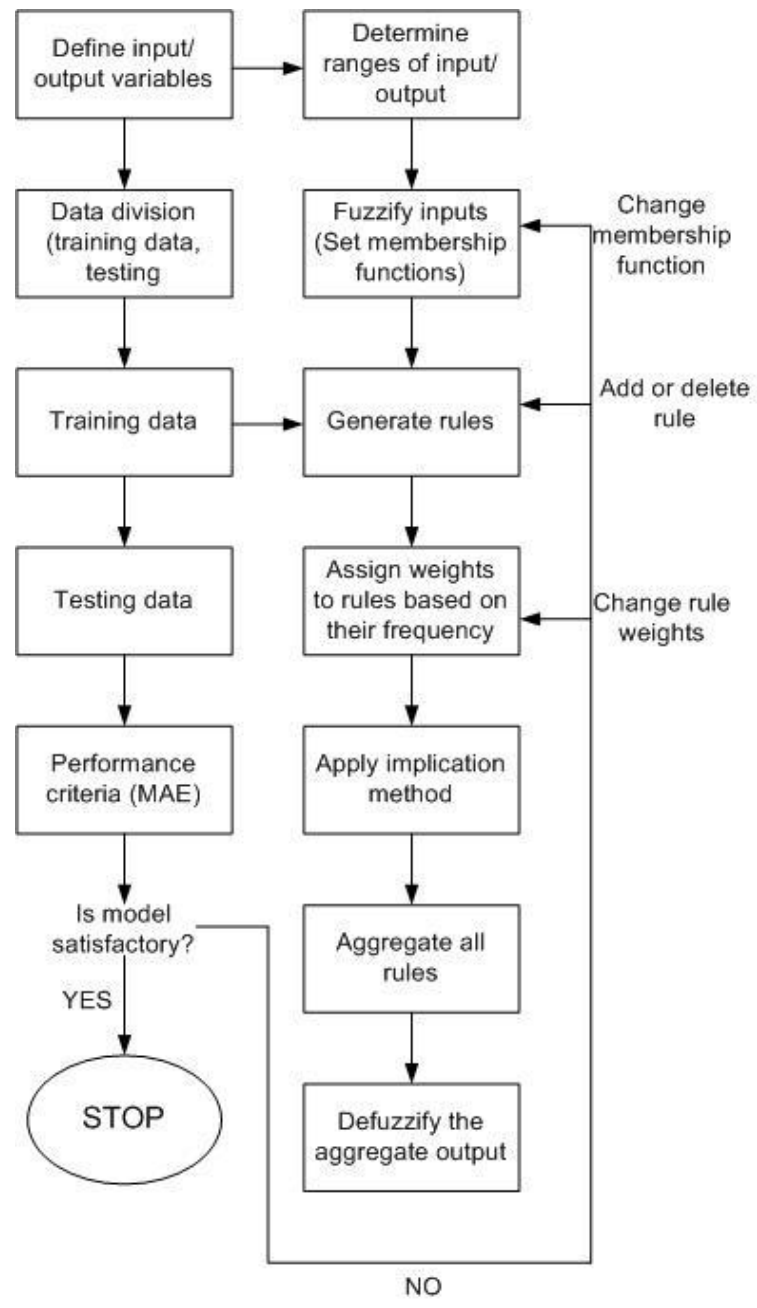

Fig. 3. Proposed fuzzy inference system flow diagram.

\section{E. Study Steps}

This study was conducted based on the following sequence of main steps as shown in Fig. 3:

Step 1. Identifying the type of fuzzy system (Mamdani or TSK type fuzzy system)

Step 2. Define the input and output variables
Step 3. Define the ranges for input and output values

Step 4. Determination of the membership functions

Step 5. Generate set of linguistic rules

Step 6. Assign rule weights.

Step 7. Aggregate all rules

Step 8. Defuzzify the aggregate output

Step 9. Validate the system.

Step 1. Identifying the type of fuzzy system: Both types of fuzzy inference system (Mamdani and TSK type) were considered and compared to each other at the end of the study.

Step 2. Defining input and output values: Input values were considered as total number of economic improvement projects and allocated budgets values at different time periods whereas output values were three types of adverse events: number of people killed, wounded, and hijacked (Fig. 4).

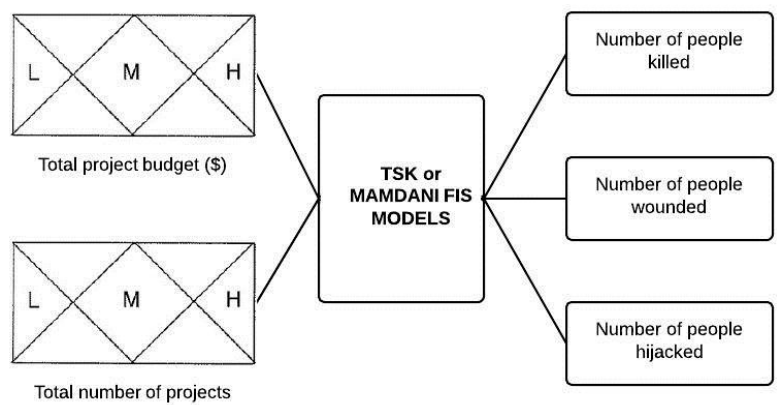

Fig. 4. General framework of FIS models used in this study.

TABLE I: INPUT VALUES AND THEIR PARAMETERS FOR BOTH FIS

\begin{tabular}{|c|c|c|c|}
\hline Input field & Range & $\begin{array}{c}\text { Linguistic } \\
\text { terms }\end{array}$ & Parameters \\
\hline $\begin{array}{l}\text { Total number } \\
\text { of aid projects }\end{array}$ & {$[0-2111]$} & $\begin{array}{l}\text { Low } \\
\text { Medium } \\
\text { High }\end{array}$ & 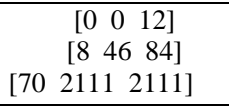 \\
\hline $\begin{array}{l}\text { Total budget } \\
\text { value }(\$)\end{array}$ & $\begin{array}{c}{[0-} \\
1,362,515,958]\end{array}$ & $\begin{array}{l}\text { Low } \\
\text { Medium }\end{array}$ & $\begin{array}{c}{\left[\begin{array}{ccc}0 & 0 & 20,000,000] \\
& \\
& {[15,000,000} \\
40,000,000 \\
65,000,000]\end{array}\right.} \\
\\
\\
\\
{[50,000,000} \\
1,362,515,958 \\
1,362,515,958]\end{array}$ \\
\hline
\end{tabular}

TABLE II: OUTPUT VALUES AND THEIR PARAMETERS FOR MAMDANI TYPE

\begin{tabular}{|c|c|c|c|}
\hline \multicolumn{4}{|c|}{ FIS } \\
\hline Output field & Range & $\begin{array}{c}\text { Linguistic } \\
\text { terms }\end{array}$ & Parameters \\
\hline $\begin{array}{l}\text { Number of } \\
\text { people killed }\end{array}$ & {$\left[\begin{array}{ll}0 & 103\end{array}\right]$} & $\begin{array}{c}\text { Very Low } \\
\text { Low } \\
\text { Medium } \\
\text { High } \\
\text { Very High } \\
\end{array}$ & $\begin{array}{c}{\left[\begin{array}{lll}0 & 0 & 1\end{array}\right]} \\
{\left[\begin{array}{lll}0.4 & 1.2 & 2\end{array}\right]} \\
{\left[\begin{array}{lll}1.5 & 4 & 6.5\end{array}\right]} \\
{\left[\begin{array}{lll}5 & 20 & 35\end{array}\right]} \\
{\left[\begin{array}{lll}30 & 103 & 103\end{array}\right]}\end{array}$ \\
\hline $\begin{array}{l}\text { Number of } \\
\text { people } \\
\text { wounded }\end{array}$ & {$\left[\begin{array}{ll}0 & 261\end{array}\right]$} & $\begin{array}{c}\text { Very Low } \\
\text { Low } \\
\text { Medium } \\
\text { High } \\
\text { Very High } \\
\end{array}$ & $\begin{array}{c}{\left[\begin{array}{lll}0 & 0 & 1\end{array}\right]} \\
{\left[\begin{array}{lll}0.5 & 1.25 & 2\end{array}\right]} \\
{\left[\begin{array}{lll}1.5 & 4.5 & 7.5\end{array}\right]} \\
{\left[\begin{array}{lll}6 & 28 & 51\end{array}\right]} \\
{\left[\begin{array}{lll}40 & 261 & 261\end{array}\right]}\end{array}$ \\
\hline $\begin{array}{l}\text { Number of } \\
\text { people } \\
\text { hijacked }\end{array}$ & {$\left[\begin{array}{ll}0 & 156\end{array}\right]$} & $\begin{array}{c}\text { Very Low } \\
\text { Low } \\
\text { Medium } \\
\text { High } \\
\text { Very High } \\
\end{array}$ & $\begin{array}{c}{\left[\begin{array}{lll}0 & 0 & 1\end{array}\right]} \\
{\left[\begin{array}{lll}0.2 & 0.9 & 1.6\end{array}\right]} \\
{\left[\begin{array}{lll}1.2 & 2.2 & 3.2\end{array}\right]} \\
{\left[\begin{array}{lll}3 & 12.5 & 22\end{array}\right]} \\
{\left[\begin{array}{lll}15 & 156 & 156\end{array}\right]}\end{array}$ \\
\hline
\end{tabular}


Step 3. Defining the ranges for input and output values: Minimum and maximum values of inputs and output fields were determined for setting relevant membership functions and parameters. The ranges, linguistic terms, and parameters for each input and output value were illustrated in Table I, II and III.

\begin{tabular}{|c|c|c|c|c|c|}
\hline \multirow{2}{*}{ Output } & \multirow{2}{*}{ Range } & \multirow{2}{*}{$\begin{array}{l}\text { Linguisti } \\
\mathrm{c} \text { terms }\end{array}$} & \multicolumn{3}{|c|}{ Parameters } \\
\hline & & & Killed & Wounded & Hijacked \\
\hline \multirow{8}{*}{$\begin{array}{c}\text { Killed } \\
\text { Wounded } \\
\text { Hijacked }\end{array}$} & \multirow{8}{*}{$\begin{array}{l}{\left[\begin{array}{ll}0 & 103\end{array}\right]} \\
{\left[\begin{array}{ll}0 & 261\end{array}\right]} \\
{\left[\begin{array}{ll}0 & 156\end{array}\right]}\end{array}$} & Lowest & {$[0]$} & {$[0]$} & [0] \\
\hline & & $\begin{array}{l}\text { Very } \\
\text { Low }\end{array}$ & [1] & [1] & [1] \\
\hline & & Low & [2] & [2] & [2] \\
\hline & & $\begin{array}{c}\text { Low } \\
\text { Medium }\end{array}$ & [3] & [3] & [3] \\
\hline & & Medium & [5] & [10] & [5] \\
\hline & & High & [20] & [30] & [10] \\
\hline & & $\begin{array}{l}\text { Very } \\
\text { High }\end{array}$ & [40] & [50] & [20] \\
\hline & & Highest & [103] & [261] & {$[156]$} \\
\hline
\end{tabular}

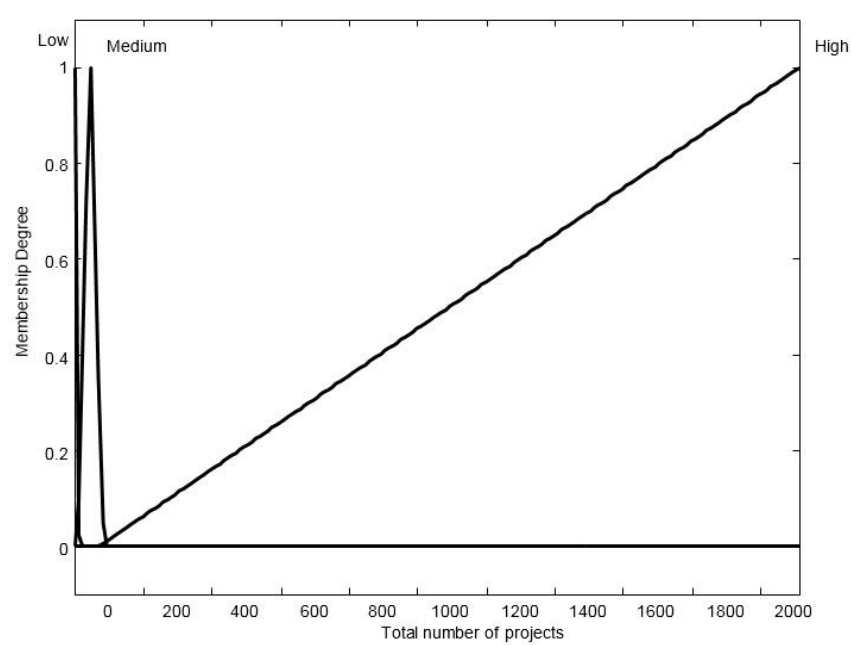

Fig. 5. Membership functions of total number of projects.

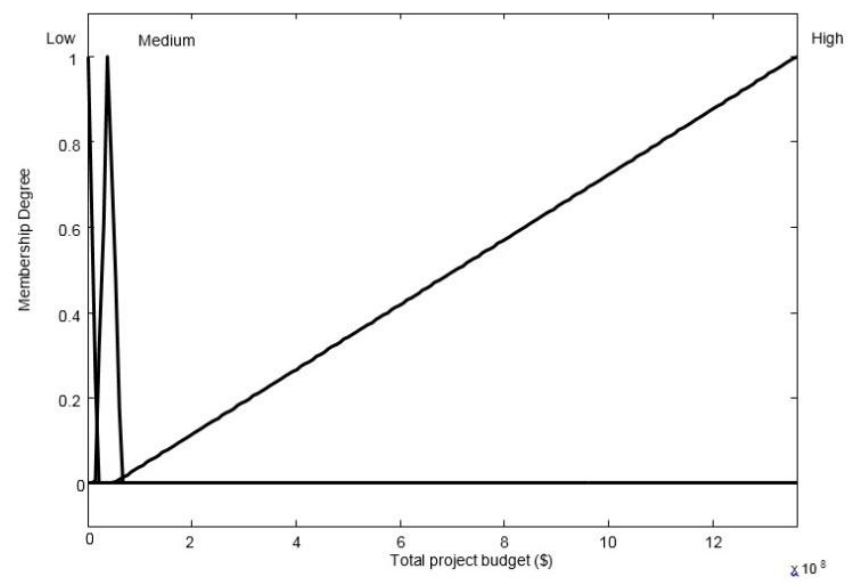

Fig. 6. Membership functions of total project budget (\$).

Step 4. Determination of the membership functions: For this study, due to its computational efficiency and ease to use, the triangular membership function was used for both input and output values. Triangular membership function for each independent and dependent variable is illustrated in Fig. 5 through 9.

Step 5. Generating set of linguistic rules: Total number of rules for each prediction methods was determined based on the number of input values and related membership functions. Training data was considered for generating IF-THEN rules.

Step 6. Assigning rule weights: The rule weights were calculated based on the number of rule frequency extracted from the training dataset and assigned to the rules. For instance the rule weight for rule $i$ was calculated as 8038 (frequency of rule $i$ ) / 38400 (total number of rules or total records in training data) $=0.209323$ (the rule weight) .

Step 7. Aggregate all rules: The output of each rule was combined to determine one fuzzy output value. For this study, this process is done by using the fuzzy "OR".

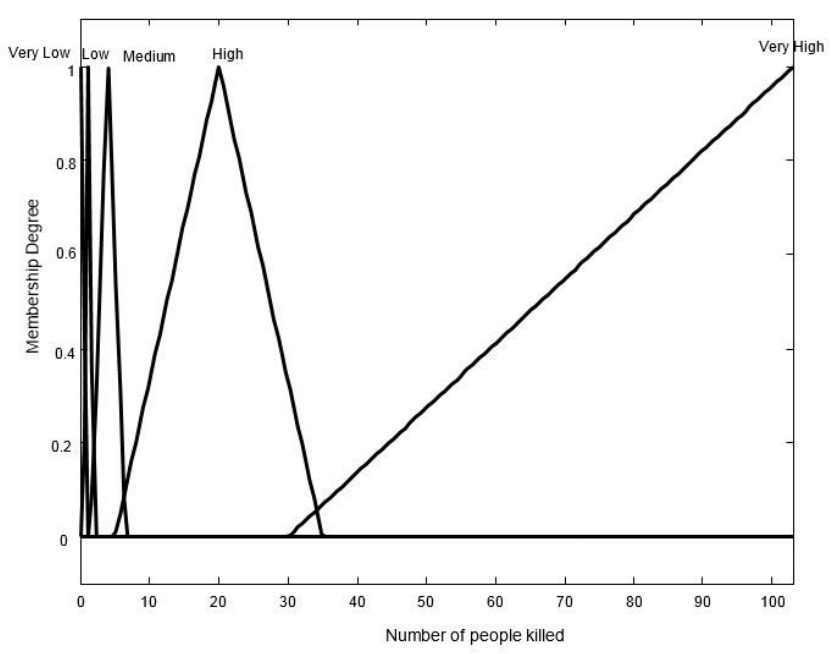

Fig. 7. Membership functions of number of people killed.

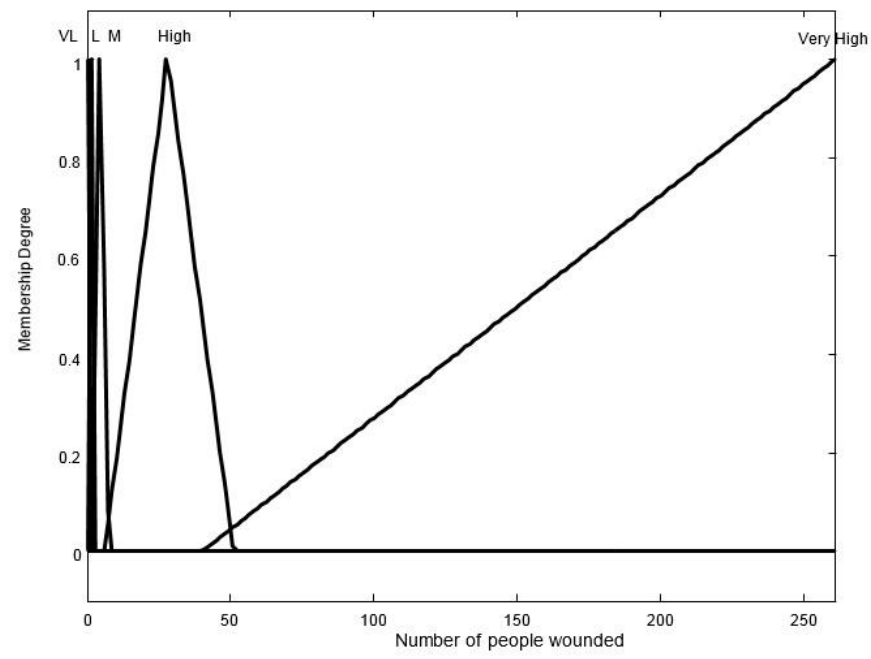

Fig. 8. Membership functions of number of people wounded.

Step 8. Defuzzify the aggregate output: For Mamdani type FIS, there are several defuzzification approaches such as Centroid of area (COA), Bisector of area (BOA), Mean of maximum (MOM), Smallest of maximum (SOM), and Largest of maximum (LOM). In this paper, we chose bisector of area (BOA) approach, since the lowest MAE values were obtained among other approaches. For the Sugeno type FIS, there are two approaches called weighted sum and weighted average. For this study, the weighted average approach was applied based on the lowest MAE values.

Step 9. Validating the system: The system was validated using testing data for year 2010. The model performance was evaluated based on the mean absolute error (MAE) value. 


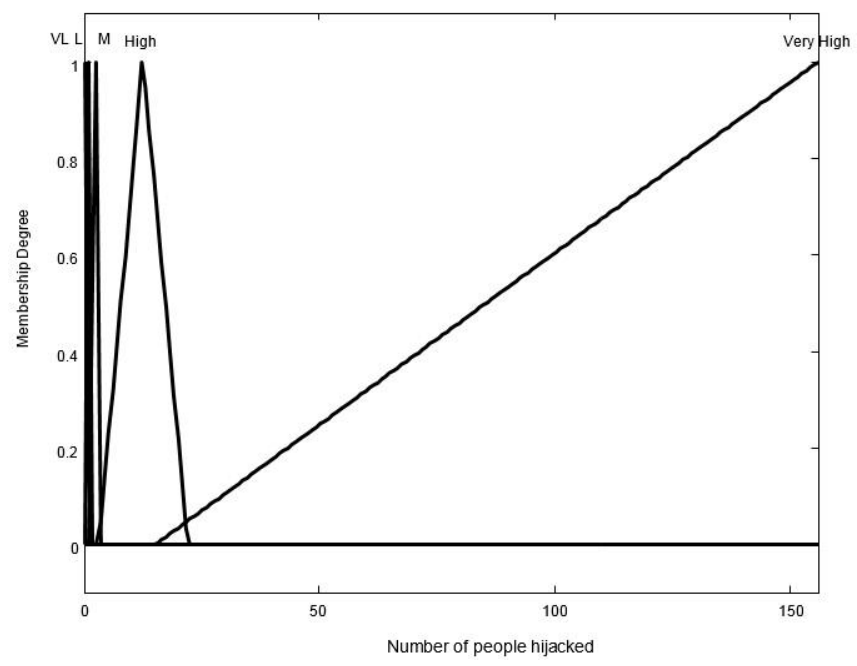

Fig. 9. Membership functions of number of people hijacked.

\section{F. Performance Metrics}

Performance metrics were used for calculating the error (difference between actual and predicted values) in the model There are several performance metrics including MSE (Mean Square Error), RMSE (Root Mean Square Error), MAE (Mean Absolute Error), and MAPE (Mean Absolute Percentage Error). In this study, the model accuracy was evaluated based on the mean absolute error (MAE) between the predicted and actual values. The following equation was used for this calculation:

$$
\mathrm{MAE}=\frac{1}{N} \sum_{i=1}^{N}\left|\left(A_{i}-P_{i}\right)\right|
$$

where $P_{i}$ and $A_{i}$ were predicted and actual values, respectively; $N$ : total number of records.

\section{RESUlTS AND DiscUSSION}

The performance values for incident types (number of people killed, wounded, and hijacked) for Mamdani and TSK models are given in Fig. 10. While the MAE values of number of killed, wounded, and hijacked output for Mamdani model were found as 0.6608, 1.0098, and 0.1981, respectively, these values were found in TSK model as $0.7907,1.1875$, and 0.2494 . The results showed that better prediction results were obtained for number of people hijacked than other dependent variables. Both Mamdani and TSK models were useful for estimating the number of adverse events based on the number of economic improvement projects and project budgets. However, Mamdani models had better predictive accuracy than TSK models as demonstrated by experimental results.

In summary, these techniques were considered to examine the relationship between adverse events and infrastructure development in an active war theater. These models may be considered as general models that used Afghanistan data for validation and verification. The model has applicability for another country looking to build infrastructure while terrorist and military activities are present.

Selecting the right fuzzy membership function and extracting of fuzzy rules are crucial and complicated for constructing fuzzy inference systems. Due to its computational efficiency and ease to use, the triangular membership function was selected to define fuzzy variables. It must be noted that this selection may not be the optimum one, and different membership functions should be tried in further studies.

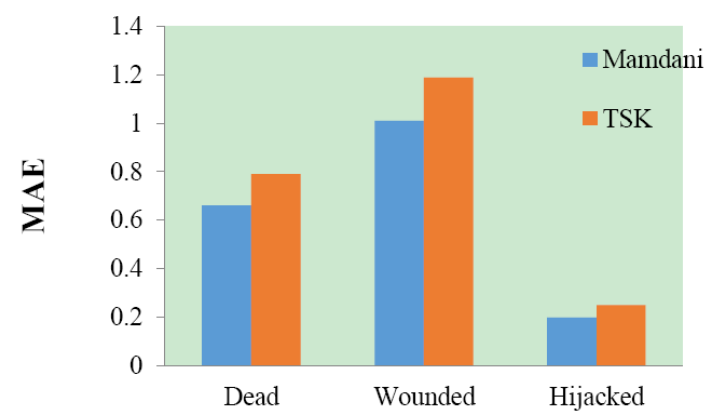

Fig. 10. Mean absolute values (MAE) of predicted values for 2010 .

The fuzzy inference rules were generated from training data and each rule was weighted based on its frequency among training records. Similarly, this approach may not be the optimum one and other approaches can be considered such as optimization-based methods (simulated annealing algorithm, genetic algorithm, etc.) for further analysis.

\section{CONCLUSIONS}

In this paper, the Takagi-Sugeno-Kang (TSK) type and the Mamdani type fuzzy inference systems (FIS) were applied to the problem of adverse events prediction based on the economic improvement projects and allocated infrastructure development budgets at different time periods in active war theater. As demonstrated by experimental results, when the model accuracy was calculated based on the mean absolute error (MAE), the Mamdani models had better predictive accuracy than the TSK models. It can be concluded that the FIS is a useful modeling technique for predicting the number of adverse events based on economic data. Such a modeling approach should be further investigated for scenario-based prediction to support decision makers in analyzing historical economic data on how regional funds allocation can best help reduce or minimize the onset of adverse events in active war theater. Since one of the main goals of this research effort was to assess the relationship between adverse events and economic infrastructure developments, in the future more input data should be used to test data patterns of such adverse events, with sensitivity analysis to study the plausible cause-and-effect relationships. Finally, geographic information systems should be implemented to improve the expected model resolution and prediction quality.

\section{ACKNOWLEDGMENT}

This study was supported in part by Grant No. [1052339], Complex Systems Engineering for Rapid Computational Socio-Cultural Network Analysis, from the Office of Naval Research. The authors acknowledge the helpful guidance of ONR program management, and the contributions of the technical team.

\section{REFERENCES}

[1] J. Clancy and C. Crossett, "Measuring effectiveness in irregular warfare," Parameters, vol. 37, no. 2, 2007.

[2] JOC, Irregular Warfare (IW) Joint Operating Concept (JOC), 2007. 
[3] Y. Bhattacharjee, "Cross-cultural research. Pentagon asks academics for help in understanding its enemies," Science, vol. 316, no. 5824, pp. 534-535, 2007.

[4] M. D. Drapeauand and B. K. Mignone, "Culture, conflict, and climate?" Science, vol. 316, no. 5831, pp. 1564-1564, 2007.

[5] HSCB Modeling Program. (2009). Available: http://www.dtic.mil/biosys/docs/HSCB-news-spring-2009.pdf

[6] J. Stanton, "Evolutionary cognitive neuroscience: dual use discipline for understanding \& managing complexity and altering warfare," in Proc. International Studies Association Conference, Portugal, 2007.

[7] C. J. Coyne and A. Pellillo, "Economic reconstruction amidst conflict: Insights from Afghanistan and Iraq," Defence and Peace Economics, vol. 22, no. 6, pp. 627-643, 2011.

[8] D. Schmorrow and D. Nicholson, Advances in Cross-Cultural Decision Making, Boca Raton: CRC Press, ch. 38, pp. 374-384, 2011.

[9] S. K. Numrich and A. Tolk, "Challenges for human, social, cultural, and behavioral modeling, " SCS M\&S Magazine, vol. 1, no. 1, 2010.

[10] D. Schmorrow, G. L. Klein, R. Foster, J. Boiney, S. Biggerstaff, P. R. Garvey, M. Koehler, and B. Costa, "Applied use of socio-cultural behavior modeling and simulation: an emerging challenge for C2," Office of The Deputy Under Secretary of Defense (Science And Technology) Rosslyn Va, 2009.

[11] G. L. Zacharias, J. MacMillan, and S. B. VanHemel, Behavioral Modeling and Simulation: From Individuals to Societies, National Academies Press, 2008.

[12] Federation of American Scientists. (2009). A GEOINT analysis of terrorism in Afghanistan, Secrecy News. [Online]. Available: http://www.fas.org/blog/secrecy/2009/05/05

[13] Open Source Center (OSC). (April 30, 2009). Afghanistan-Geospatial analysis reveal patterns in terrorist incidents 2004-2008. [Online]. Available: http://www.fas.org/irp/dni/osc/afghan-geospat.pdf

[14] L. A. Zadeh, "Fuzzy logic, neural networks and soft computing," Communications of the ACM, vol. 37, no. 3, pp. 77-84, 1994.

[15] D. Nauck, F. Klawonn, and R. Kruse, Foundations of Neuro-Fuzzy Systems, John Wiley \& Sons, Inc., New York, NY, USA, 1997.

[16] U. Inyaem, P. Meesad, C. Haruechaiyasak, and D. Tran, "Terrorism event classification using fuzzy inference systems," International Journal of Computer Science and Information Security, vol. 7, no. 3, 2010.

[17] E. Çakıt, "Investigating the relationship between adverse events and infrastructure development in an active war theater using soft computing techniques," Ph.D. dissertation, Dept. Industrial Eng. and Management Systems, University of Central Florida, Orlando, Florida, 2013.

[18] S. N. Sivanandam, S. Sumathi, and S. N. Deepa, Introduction to Fuzzy Logic Using MATLAB, Springer, 2008

[19] H. J. Zimmermann, Fuzzy Set Theory - and Its Applications, $3^{\text {rd }}$ edition, Kluwer Academic Publishers, 1996.

[20] A. Haji and M. Assadi, "Fuzzy expert systems and challenge of new product pricing," Computers \& Industrial Engineering, vol. 56, no. 2, pp. 616-630, 2009.
[21] E. H. Mamdani and S. Assilian, "An experiment in linguistic synthesis with a fuzzy logic controller," International Journal of Man-Machine Studies, vol. 7, no. 1, pp. 1-13, 1975.

[22] J. S. R. Jang, C. T. Sun, and E. Mizutani, Neuro-Fuzzy and Soft Computing: A Computational Approach to Learning and Machine Intelligence, Upper Saddle River, NJ: Prentice Hall, 1997.

[23] T. Takagi and M. Sugeno, "Fuzzy identification of systems and its applications to modeling and control," IEEE Transactions on Systems, Man and Cybernetics, vol. 1, pp. 116-132, 1985.

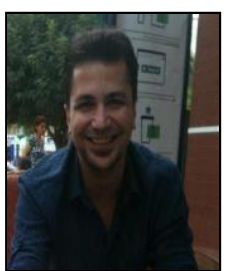

Erman Cakit received his $\mathrm{PhD}$ degree in industrial engineering from the University of Central Florida, Orlando, USA, in 2013. He is currently working as an assistant professor at the Department of Industrial Engineering, Aksaray University, Turkey. His research interests include applications of human factors / ergonomics, cross cultural decision making, human social culture behavior (HSCB) modeling using soft computing, applied statistics, and geographic information systems. He is a member of IACSIT, SCIEI and IEEE Computational Intelligence Society.

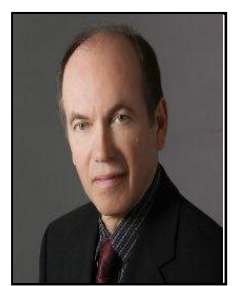

Waldemar Karwowski is a professor and the chairman of the Department of Industrial Engineering and Management Systems. He is also an executive director of Institute for Advanced Systems Engineering, University of Central Florida, Orlando, USA. He holds an M.S. (1978) in production engineering and management from the Technical University of Wroclaw, Poland, and a Ph.D. (1982) in industrial engineering from Texas Tech University. He was awarded D.Sc. degree in management science by the State Institute for Organization and Management in Industry, Poland (2004). He is the past president of the International Ergonomics Association (2000-2003), and the past president of the Human Factors and Ergonomics Society (2007). He served on the Committee on Human Factors/Human Systems Integration, National Research Council, the National Academies, USA (2007-2011). He is the author or coauthor of over 380 scientific publications which focus on occupational ergonomics, human systems integration, human-computer interaction, neuro-fuzzy modeling, nonlinear dynamics of human performance, and neuroergonomics. He is a member of IEEE/Systems, Man \& Cybernetics Society and a fellow member of the Human Factors and Ergonomics Society. 